\title{
Observations of Gas-Phase Atoms and Molecules
}

\author{
John H. Lacy \\ Dept. of Astronomy, Univ. of Texas, Austin, TX 78712, USA
}

\begin{abstract}
Some recent observations of interstellar, circumstellar, and stellar atoms and molecules, with ground-based telescopes and ISO, are discussed. The advantages of observations with SIRTF and SOFIA, and needed laboratory work are mentioned more briefly.
\end{abstract}

\section{Interstellar Atoms and Ions}

The mid and far-infrared ionic fine-structure lines are very important probes of ionized gas, especially in optically obscured regions. Ions with $p^{1}\left({ }^{2} \mathrm{P}\right), p^{2}$ $\left({ }^{3} \mathrm{P}\right), p^{4}\left({ }^{3} \mathrm{P}\right)$, and $p^{5}\left({ }^{2} \mathrm{P}\right)$ electron configurations have fine-structure transitions. About half of these lines are observable from the ground, and most of the rest are observable from airborne telescopes, but the complete set only became observable with ISO. These lines are likely to be among the most important lines for SIRTF IRS observations. The ISO SWS spectra of IRc2 in Orion (van Dishoeck et al. 1998) and Sgr A West (Lutz et al. 1996) show many of the hydrogen recombination and ionic fine-structure lines.

Frequencies of the atomic and ionic lines are generally well known, often through astronomical observations (Kelly \& Lacy 1995, Feuchtgruber et al. 1997). Collisional excitation coefficients and recombination coefficients are probably accurate enough for the $2 \mathrm{p}$ ions, but not so accurate for the $3 \mathrm{p}$ and heavier ions.

\section{Interstellar Molecules}

Infrared spectroscopy of interstellar molecules has been most important for observations of light and symmetric molecules, which have no millimeter-wave transitions. Ground-based observations include $\mathrm{H}_{2}$ (Gautier et al. 1976; Beck et al. 1982), $\mathrm{CO}$ (Scoville et al. 1983), $\mathrm{C}_{2} \mathrm{H}_{2}, \mathrm{CH}_{4}$, and $\mathrm{HCN}$ (Lacy et al. 1989, 1991), and $\mathrm{H}_{3}^{+}$(Geballe \& Oka 1996). In addition to more extensive observations of these molecules, ISO was used to observe HD (Wright et al. 1999; Bertoldi et al. 1999), $\mathrm{CH}_{3}$ (Feuchtgruber et al. 2000), $\mathrm{H}_{2} \mathrm{O}$ (Helmich et al. 1996), $\mathrm{CO}_{2}$ (Boonman et al. 2000), and $\mathrm{C}_{3}$ (Cernicharo et al. 2000). Most infrared observations of interstellar molecules are in absorption toward pre-main-sequence stars, which serve as bright background sources. As a result, these observations probe the chemistry of star-forming regions. In addition, many of the molecules which have been observed in the infrared are either formed in icy grain mantles or stored there, and released into the gas phase when the grains are heated by a young star, making them useful probes of the influence of grain mantles on gas-phase abundances. 
Compared to the ground-based telescopes, ISO had the advantage of being above the Earth's atmosphere, but it had rather limited resolution, with the SWS $\mathrm{R}=2000$ higher sensitivity mode used for most observations. SIRTF will have excellent sensitivity, but even lower resolution $(R \approx 600)$. Much higher resolution spectrographs will be available on SOFIA and ground-based telescopes, with $\mathrm{R}=100,000$ and higher planned. Although observations from these platforms will be limited by telluric absorption, higher resolution will allow accurate abundance determinations as well as the extraction of kinematic information from line profiles.

For most of the promising molecules, line frequencies are generally well known. Helmich (1996) provides a convenient compilation. Band strengths are generally known for the stable molecules, but often not for the radicals. Probably the most important laboratory data for understanding infrared observations of interstellar molecules are reaction rates, which are needed for chemical models. Information about ice-mantle reactions are particularly important.

\section{Protoplanetary Disks}

Ground-based infrared observations of circumstellar disks have concentrated on the dust continuum. The only infrared observations of gas are of $\mathrm{CO}$, mostly through its $2 \mu \mathrm{m}$ overtone (Carr \& Tokunaga 1992, Chandler et al. 1993). These observations probe gas at $\ll 1 \mathrm{AU}$, where temperatures and densities are high enough to populate high $\mathbf{J}$ and $\mathbf{v}$ levels. Millimeter observations probe much cooler gas, at $\gg 1 \mathrm{AU}$, but the gas in the planet-forming region, $\sim 1-10 \mathrm{AU}$, has been difficult to study.

Mid-infrared observations may change this. $\mathrm{H}_{2}$ rotational-line emission was seen with ISO from the GG Tau disk (Thi et al. 1999). These lines, as well as mid-infrared lines from other molecules, are excited at temperatures $\sim 300 \mathrm{~K}$. SOFIA will have mid-infrared spectrographs with sufficient resolution $\left(\sim 3 \mathrm{~km} \mathrm{~s}^{-1}\right)$ to use line profiles to map the distribution of the emitting gas.

As with the study of interstellar molecules, laboratory data are needed more to support modeling of disks than to support the observations directly. Laboratory work is needed to provide data on reaction rates, collisional transition rates (both vibrational and rotational), condensation and evaporation of ices, formation, growth, and destruction of refractory grains, and gas dynamical processes such as viscosity and convection that drive inflow and mixing in disks.

\section{Circumstellar Molecules}

Molecules in outflows around AGB and post-AGB stars have been studied for some time. For example, various hydrocarbons were observed in IRC +10216 (Ridgway et al. 1976), and SiO was observed in VY CMa (Geballe, Lacy \& Beck 1979). Observations with ISO added to the number of molecules observed, including $\mathrm{CO}_{2}, \mathrm{H}_{2} \mathrm{O}$, and $\mathrm{OH}$ (Justtanont et al. 1996), and polyacetylenes (Cernicharo et al. 1996). However, the ISO observations were mostly made with $\mathrm{R}=2000$, and the higher resolution ground-based observations show P-Cygni profiles. As a result, observations which do not separate the emission and absorption components of the lines are of limited quantitative value. SIRTF spec- 
troscopy will be even more hampered by low resolution. SOFIA will be unable to observe $\mathrm{CO}_{2}$ and will be limited by the Earth's atmosphere for observations of some other molecules, but should be able to measure excellent spectra of these very bright sources at the wavelengths where it can work.

Probably the two main types of laboratory data that are needed are collisional excitation rates and information on dust condensation. Modeling of the P-Cygni line profiles will require calculations of both radiative and collisional transitions, as the lines are formed in the region of the outflows where vibrational populations are falling out of LTE. The observations should provide information on dust condensation, as some of the grain precursor molecules are the most easily observed: $\mathrm{C}_{2} \mathrm{H}_{2}$ for graphitic grains, and $\mathrm{SiO}$ for silicate grains.

Acknowledgments. This work was supported by grants from USRA, the Texas Advanced Research Program, and the Rom Rhome International Professional Development Fund.

\section{References}

Beck, S. C., et al. 1982, ApJ, 253, L83

Bertoldi, F., et al. 1999, A\&A, 346, 267

Boonman, A. M. S., et al. 2000, in ISO Beyond the Peaks, eds. A. Salama et al. (Noordwijk: ESA), 67

Carr, J. S. \& Tokunaga, A. T. 1992, ApJ, 393, L67

Cernicharo, J., et al. 1996, A\&A, 315, L201

Cernicharo, J., et al. 2000, ApJ, 534, L199

Chandler, C. J., et al. 1993, ApJ, 412, L71

Feuchtgruber, H., et al. 1997, ApJ, 487, 962

Feuchtgruber, H., et al. 2000, ApJ, 535, L111

Gautier, T. N., et al. 1976, ApJ, 207 L129

Geballe, T. R., et al. 1979, ApJ, 230, L47

Geballe, T. R. \& Oka, T. 1996, Nature, 384, 334

Helmich, F. P. 1996, Ph.D. thesis, University of Leiden

Helmich, F. P., et al. 1996, A\&A, 315, L173

Justtanont, K., et al. 1996, A\&A, 315, L217

Kelly, D. M. \& Lacy, J. H. 1995, ApJ, 454, L161

Lacy, J. H., et al. 1989, ApJ, 342, L43

Lacy, J. H., et al. 1991, ApJ, 376, 556

Lutz, D., et al. 1996, A\&A, 315, L269

Ridgway, S. T., et al. 1976, Nature, 264, 345

Scoville, N. Z., et al. 1983, ApJ, 275, 201

Thi, W.-F., et al. 1999, A\&A, 350, 541

van Dishoeck, E. F., et al. 1998, ApJ, 502, L173

Wright, C. M., et al. 1999, ApJ, 515, L29 